\title{
EVALUATION OF IN VITRO AND IN VIVO EQUINE MICROBIAL POPULATION SHIFTS IN RESPONSE TO COLIC CONDITIONS \\ Erin Venable
} 13 effects on VFA and $\mathrm{NH}_{3}$ concentration, and $\mathrm{pH}$ following starvation or starch overload.

\author{
Dr. Monty Kerley, Dissertation Supervisor
}

Abstract

We designed two continuous culture experiments to test the effects of starch dosage and starvation upon equine fecal microflora. Diets were designed containing fructooligosaccharides and mannanoligosaccharides in order to investigate treatment This research indicated that total volatile fatty acid (VFA) concentration may be affected by treatment following starch overload. Additionally, branched chain VFA, and butyric acid concentrations were different across treatment in both experiments. This research indicated that following an abrupt change in the starch content of the diet, or a starvation period, those effects may be ameliorated by diet. In our third experiment, we performed pyrosequencing in order to phylogenetically characterize the shifts in the microbial populations observed in the feces of eight horses collected from both the colic (large intestinal-nonsurgical) and healthy states. Healthy samples were collected by the attending/referring veterinarian 60 days ( \pm 30) post discharge. DNA was extracted from the fecal samples and the V1, V2, and V3 region of the 16S rDNA was amplified via emulsion PCR.

5 When ubiquitous microbes were ranked colic > healthy, four were significant. All sick horses had greater reads of Clostridium phytofermentans, an uncultured Clostridiales bacterium, and Bacteroidetes as well as an uncultured bacteroidetes bacterium. These data suggest there is a need for broad bacterial diversity in the healthy equine gut. 